\title{
Evaluation of the Possible Neurotoxic Effect of the Bone Cement on the Facial Nerve: An Experimental Study
}

\author{
Numan Kökten ${ }^{1} \cdot$ Oğuz Kadir Eğilmez ${ }^{2}$ M. Tayyar Kalcıoğlu' $\cdot$ Mustafa Baran³ $\cdot$ A. Işın Doğan Ekici ${ }^{4}$ \\ ${ }^{I}$ Department of Otorhinolaryngology, Goztepe Training and Research Hospital, Istanbul Medeniyet University, Faculty of Medicine, Istanbul; \\ ${ }^{2}$ Department of Otorhinolaryngology, Sakarya University Training and Research Hospital, Sakarya; \\ ${ }^{3}$ Department of Physiology, Istanbul Medeniyet University, Faculty of Medicine, Istanbul; \\ ${ }^{4}$ Department of Pathology, Yeditepe University, Faculty of Medicine, Istanbul, Turkey
}

Objectives. To investigate neurotoxic effect of bone cement (BC) on facial nerve by using electrophysiological and histopathological methods.

Methods. This study included 20 male albino Wistar rats, divided into four equal groups. Group A was designed as the control group, while group B was sham group. In the group C, BC solution was dropped onto the facial nerve trunks of rats and washed with physiological saline after 5 seconds. In the group D, BC solution was dropped onto the facial nerve trunks of rats and after allowing 5 minutes to dry, wounds were closed. Pre- and postoperative (on 4th week) evoked electromyography (EMG) measurements were done. For histopathological assessments, the rats were euthanized and tissue samples of facial nerve and surrounding areas were collected.

Results. According to the wave amplitude levels of evoked EMG, postoperative amplitude levels of group D were significantly decreased, compared to preoperative amplitude levels $(P=0.043)$. We found no statistically significant difference in inflammation among the groups. In none of the groups, foreign body reaction and granulation tissue were not detected in any of the groups. In addition, degeneration in axon, myelin, or perineural nets was not detected in any of the groups.

Conclusion. This study results suggest that $\mathrm{BC}$ has no direct toxicity on facial nerve, while it has indirect effects, by decreasing amplitude. Therefore, we conclude that direct contact of $\mathrm{BC}$ with nerve should be avoided, and the area should be cleaned by aspiration or washing with physiological saline in case of contact.

Keywords. Bone Cements; Rats; Electromyography; Neurotoxicity; Facial Nerve

\section{INTRODUCTION}

Bone cement (BC) was developed in 1970s by two chemists named Alan Wilson and Brian Kent. BC is a polymer compound

\footnotetext{
- Received July 12, 2017

Revised November 24, 2017

Accepted January 9, 2018

- Corresponding author: M.Tayyar Kalcioğlu

Department of Otorhinolaryngology, Goztepe Training and Research

Hospital, Istanbul Medeniyet University, Faculty of Medicine, Dr. Erkin

Caddesi. 34722, Kadıköy, Istanbul, Turkey

Tel: +90-216-5709074, Fax: +90-212-2874002

E-mail: mtkalcioglu@hotmail.com
}

that can bond bone, metallic, ceramic and synthetic materials together. BC is formed by a reaction between organic polyacrylic acid and inorganic aluminum ionomer glass particles containing alkali aluminum [1]. BC has been safely used in dentistry and orthopedic surgery for long time periods; also, it is becoming more widespread for craniofacial reconstructive surgery and neurosurgery [1-3].

The use of $B C$ in middle ear surgery was first reported by Geyer and Helms in 1990 [2]. It is used in otorhinolaryngologic surgery, particularly in middle ear ossicular reconstruction, such as repairing incudostapedial joint weakness or incus long arm defects, piston stabilization in stapedotomy or stapedectomy surgeries,

Copyright $\odot 2018$ by Korean Society of Otorhinolaryngology-Head and Neck Surgery.

This is an open-access article distributed under the terms of the Creative Commons Attribution Non-Commercial License (http://creativecommons.org/licenses/by-nc/4.0)

which permits unrestricted non-commercial use, distribution, and reproduction in any medium, provided the original work is properly cited. 
repair of the external ear canal wall, reduction of mastoid cavity, stabilization of cochlear implant electrodes and implantable hearing devices, repair of the cerebrospinal fluid fistula or semicircular canal fistula, repair of petrous bone defects related to acoustic neuroma surgery, skull base, and dural defects [1-4].

The most important disseminative features of $\mathrm{BC}$ are inertness, biocompatibility, accepted as safe, ease of use in difficult areas, low-cost, and minimal rejection rate [3]. In the literature, there are several case reports and clinical trials that reported BC-related inflammation, cytotoxicity, granulation formation, neurotoxicity, transient facial paralysis, subacute myoclonic encephalopathy and subacute lethal aluminum encephalopathy, which can limit the use of BC [1,5-11]. However, there have been no systematic studies on the possible neurotoxic effects of $\mathrm{BC}$ on facial nerve.

Although the facial nerve is located in a bone canal in the temporal bone, congenital or acquired dehiscent may occur. For that reason, it should be kept in mind that the use of chemicals like BC for middle ear surgeries may cause chemical-related damages. Well-planned and objective studies towards BC-related neurotoxicity are insufficient. Studies that reported neurotoxicity were usually case reports. One of the experimental studies, beagles that were placed with intracranial parietal and suboccipital BC were employed, and BC-related neurotoxicity was investigated by using auditory brain response test. Although test results were not significantly different, gliosis in the cerebral cortex and acoustic nerves were detected [12]. And in one thesis study, BC-related neurotoxicity was investigated by using rabbit sciatic nerve and they reported that, although $\mathrm{BC}$ might not have a direct effect on nerve, it has increased the toxicity of the surrounding tissue and induced granulation tissue and foreign body reaction [13]. To the best of our knowledge, there is no objective study which investigates the effects of $\mathrm{BC}$ on facial nerve. Therefore, in this study, we aimed to investigate neurotoxic effect of $\mathrm{BC}$ on the facial nerve of rats by using electrophysiological and histopathological methods.

\section{MATERIALS AND METHODS}

This study was approved by the local Ethics Committee on ani-

\section{H I G G H L I G G H T S}

- In this study, it was shown that bone cement has no direct toxic effect on facial nerve.

- None of the animals demonstrated facial paralysis and nerve conduction blocks.

- Histopathological examination showed no degeneration in axon, myelin or perineural nets.

- Minimal inflammation and myositis were observed. mal experiments (protocol No. 01.11.2016-503). In the study, 20 male Wistar rats aged between 12 and 14 weeks and weighing between 250 and $300 \mathrm{~g}$ were used. AquaCem (Dentsply, Konstanz, Germany) was used in this study as a BC source. According to the provider, 1,000 mg AquaCem powder contains $744.14 \mathrm{mg}$ calcium-sodium-fluoro-phosphoroaluminium-silicate (21:2:15:2:14:46), $247.68 \mathrm{mg}$ polyacrylic acid, $8.10 \mathrm{mg}$ tartaric acid and yellow ferric oxide (E 172). To obtain polymer cement, one measure of powder was mixed with two drops of water in a container and mixed for 2-3 minutes. After the beginning of the solidification of liquefied BC, enough quantity of that solution was taken from the container and applied to the study area. So, it was aimed to preserve the tissues from the heat and prevent thermal injury to the facial nerve.

Experiments were performed only on left facial nerves and evoked electromyography (EMG) records were also taken on left nerves. Rats were given general anesthesia pre- and postoperatively on the fourth week, by intramuscular ketamine hydrochloride $(50 \mathrm{mg} / \mathrm{kg})$ and xylazine hydrochloride $(5 \mathrm{mg} / \mathrm{kg})$ and the rectal body temperature of rats was between $34.5^{\circ} \mathrm{C}$ and $36.5^{\circ} \mathrm{C}$. To obtain compound muscle action potential (CMAP) from the mandibular branch of the facial nerve, evoked EMG was recorded by using PowerLab 26T multiple recording devices and LabTutor software (ADInstruments, New South Wales, Australia). Amplitude, duration, latency, and supramaximal stimuli thresholds were recorded and evaluated between experimental and control groups.

The evoked EMG recordings were obtained according to a model which was described by Salomon et al. [14]. During evoked EMG records, bipolar subdermal needle electrodes were used for stimulation and recording. These bipolar electrodes were formed specifically by using monopolar electrodes that had a thickness of $0.35 \mathrm{~mm}$ and length of $12 \mathrm{~mm}$. To form, monopolar electrodes were placed $5 \mathrm{~mm}$ apart from each and taped with insulated bands. Also, for subcutaneous entry, 3-mm gap were left open at the tip while 9-mm part taped for insulation. Monopolar needle electrode was used for static grounding which has 3-mm gap for subcutaneous entry. The recording electrode was placed subliminally, positioned $3 \mathrm{~mm}$ away from the corner of the mouth and parallel to the lower lip, and the stimulation electrode positioned $25 \mathrm{~mm}$ away from the corner of the mouth to stimulate the main trunk of the facial nerve. The anode $(+)$ was set at the proximal, and cathode $(-)$ at the distal position. The static grounding electrode was placed subdermally, between recording and stimulation electrodes. For electrical stimulation, stimuli were given starting from $0.5 \mathrm{~mA}$ and increasing up to $5 \mathrm{~mA}$ at $0.5 \mathrm{~mA}$ increments. For each stimulus, duration was $50 \mathrm{~ms}$. The duration between stimulus and the first wave appeared on the isoelectric line was determined as latency (in millisecond). The time between the start and end of wave was calculated as wave duration (in millisecond). The distance between the positive and negative waves (in millivolt) was deter- 
mined as amplitude. Supramaximal stimulation thresholds were determined as the level that stimulate of all fibers. To compare records in a constant value, only records that obtained under 2.5 $\mathrm{mA}$ stimulus were evaluated. And this value was the closest value to the average supramaximal stimulus threshold.

Control group (group A) consisted of five rats without any intervention, only evoked EMG values were measured on these rats. For the rest of the animals, postauricular region was entered subcutaneously by $2 \mathrm{~cm}$ skin incision, trapezius muscle tendons were moved slightly to the lateral with blunt dissection. The trunk of the facial nerve rising from the stylomastoid foramen was located and branches through parotid gland were followed. Group B was planned as sham and rats on this group were operated to find the facial nerve and their wounds were closed without any additional intervention, by using $4 / 0$ vicryl. $\mathrm{BC}$ solution was prepared as described before, 1-2 drops of distilled water mixed with $\mathrm{BC}$ powder and thickened by using elevator. Animals in the group $\mathrm{C}$, received $\mathrm{BC}$ solution on their facial nerves, solution was dropped to the nerve trunks (area before the nerve branches) and kept for 5 seconds (during hearing reconstruction, $\mathrm{BC}$ occasionally flows from the affected area and contacts the surrounding tissues. In such cases, it is cleared within seconds of the current location. In group $\mathrm{C}$, we intended to simulate this short-time interaction condition, and for this reason we applied BC for 5 seconds and cleaned it from the tissue). Then, wounds were closed after solution was washed with serum physiologic. In the group D, BC solution was dropped onto the same area, and kept for 5 minutes drying. Then, the operation was terminated without cleaning the $\mathrm{BC}$ solution and wounds were closed (Fig. 1). In addition, after the operations, activity in whiskers in all animals was checked daily.

Postoperative evoked-EMG measurements from all groups were taken on the fourth week, under anesthesia. Following evoked EMG, rats were euthanized by guillotine decapitation.

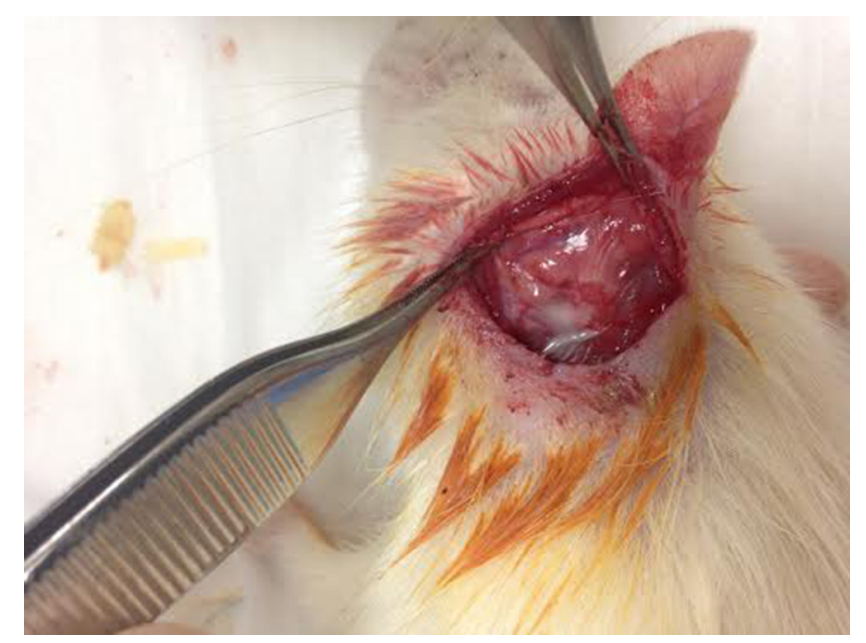

Fig. 1. Group D: bone cement was dropped and kept for 5 minutes drying.
For histopathological assessments, facial nerve (1-cm long, before the nerve branches) with surrounding tissue samples was collected and embedded in paraffin blocks after fixation with formaldehyde solution (10\%) for 24 hours. Sections of 4-6 $\mu \mathrm{m}$ thickness were obtained by using a microtome, and stained with hematoxylin and eosin and S-100 immunohistochemistry stain (Biocare Medical, Pacheco, CA, USA). Slides then were evaluated by using light microscope (Olympus BX53, Tokyo, Japan) and images were taken under $\times 40, \times 100$, and $\times 200$ magnifications. Micro-measurements were quantified by using Olympus DP73 camera and digital microscopy software and all parameter were classified individually. Based on histopathological assessments, the changes were grouped as foreign body reaction (no, yes), granulation tissue (no, yes), inflammation ( $\mathrm{n}=0$; mild, + ; moderate, ++; extensive, +++; widespread inflammation and necrosis, ++++ ; we set the amount of cells below 10 as none, $10-30$ as mild, $30-60$ as moderate, $60-100$ as extensive and above 100 as widespread), axonal degeneration (no, yes), status of the perineural nets and myelin structure (myelin structure preserved, irregular myelin structure). All samples were evaluated by the same pathologist blindly.

To evaluate test results, pre- and postoperative means and standard deviations for wave duration, latencies, supramaximal stimulus thresholds and amplitudes were calculated in the study and control groups. Histopathological data were evaluated with statistical results. For statistical analysis, IBM SPSS ver. 22.0 (IBM Corp., Armonk, NY, USA) was used. The Kruskal-Wallis test was used for the comparison between groups and Wilcoxon signed-rank test was used for dependent groups. A $P$-value $<0.05$ was considered statistically significant with $95 \%$ confidence interval.

\section{RESULTS}

According to the wave amplitude levels of the evoked-EMG parameter, postoperative amplitude levels of group D were significantly decreased compared to preoperative amplitude levels $(P<0.05, P=0.043)$. In terms of pre- and postoperative amplitude levels, there were no significant alterations in the other groups $(P>0.05)$ (Table 1$)$.

Based on the levels of evoked-EMG wave latency, wave duration and supramaximal stimulus thresholds were compared, and no statistically significant difference was found between groups for pre- and postoperative measurements $(P>0.05)$ (Table 1$)$. The CMAP results of pre- and postoperative evoked EMG measurements are shown in Tables 2 and 3.

During the tissue collection for histopathological assessment, no trace of $\mathrm{BC}$ was observed in the rats of group $\mathrm{D}$. BC was considered to dissolve in tissue by ionization.

During the histopathological evaluation, no statistically significant difference was observed between groups in terms of in- 
Table 1. The postoperative-preoperative difference of values (amplitude, latency, duration, and supramaximal intensity level)

\begin{tabular}{lcccccc}
\hline Variable & Group B & $P$-value & Group C & $P$-value & Group D & $P$-value \\
\hline Amplitude $(\mathrm{mV})$ & $0.13 \pm 1.33$ & 0.893 & $0.24 \pm 2.44$ & 0.893 & $-1.67 \pm 0.91$ & 0.043 \\
Latency $(\mathrm{ms})$ & $0.32 \pm 0.29$ & 0.068 & $-0.28 \pm 0.48$ & 0.197 & $-0.28 \pm 0.48$ & 0.465 \\
Duration (ms) & $0.62 \pm 0.43$ & 0.138 & $-0.04 \pm 0.51$ & 0.892 & $0.10 \pm 0.91$ & 0.500 \\
Stimulus intensity (mA) & $0.80 \pm 0.57$ & 0.066 & $-0.60 \pm 0.96$ & 0.197 & $0.80 \pm 0.76$ & 0.066 \\
\hline
\end{tabular}

Values are presented as mean \pm standard deviation. Group B (sham), group C (bone cement [BC] was applied for 5 seconds and cleaned), and group D (BC was applied and kept for 5 minutes drying).

a)Wilcoxon signed-rank test.

Table 2. The preoperative CMAP values of amplitude, latency, duration and supramaximal intensity level

\begin{tabular}{lccccc}
\hline Variable & Group A & Group B & Group C & Group D & $P$-value ${ }^{\text {a) }}$ \\
\hline Preoperative amplitude $(\mathrm{mV})$ & $2.49 \pm 1.38$ & $2.23 \pm 1.01$ & $2.71 \pm 1.58$ & $3.71 \pm 1.30$ & 0.326 \\
Preoperative latency $(\mathrm{ms})$ & $1.66 \pm 0.31$ & $1.46 \pm 0.23$ & $1.74 \pm 0.48$ & $1.34 \pm 0.27$ & 0.606 \\
Preoperative duration (ms) & $3.56 \pm 0.86$ & $3.68 \pm 0.75$ & $3.84 \pm 0.48$ & $3.70 \pm 0.29$ & 0.904 \\
Preoperative stimulus intensity (mA) & $2.50 \pm 0.50$ & $2.80 \pm 0.76$ & $3.00 \pm 1.06$ & $1.60 \pm 0.55$ & 0.082 \\
\hline
\end{tabular}

Values are presented as mean \pm standard deviation. Group A (control), group B (sham), group C (bone cement [BC] is applied for 5 seconds and cleaned), and group D (BC was applied and kept for 5 minutes drying).

CMAP, compound muscle action potential.

a)Kruskal-Wallis Test.

Table 3. The postoperative CMAP values of amplitude, latency, duration and supramaximal intensity level

\begin{tabular}{lccccc}
\hline Variable & Group A & Group B & Group C & Group D & $P$-value ${ }^{\text {a) }}$ \\
\hline Postoperative amplitude $(\mathrm{mV})$ & $2.49 \pm 1.38$ & $2.36 \pm 1.42$ & $2.95 \pm 1.20$ & $2.04 \pm 0.99$ & 0.728 \\
Postoperative latency $(\mathrm{ms})$ & $1.66 \pm 0.31$ & $1.78 \pm 0.37$ & $1.46 \pm 0.09$ & $1.54 \pm 0.33$ & 0.230 \\
Postoperative duration (ms) & $3.56 \pm 0.86$ & $3.90 \pm 0.59$ & $3.80 \pm 0.32$ & $3.80 \pm 0.76$ & 0.623 \\
Postoperative stimulus intensity (mA) & $2.50 \pm 0.50$ & $2.60 \pm 0.82$ & $2.40 \pm 1.29$ & $2.40 \pm 0.96$ & 0.952 \\
\hline
\end{tabular}

Values are presented as mean \pm standard deviation. Group A (control), group B (sham), group C (bone cement [BC] is applied for 5 seconds and cleaned), and group D (BC was applied and kept for 5 minutes drying).

CMAP, compound muscle action potential.

a)Kruskal-Wallis Test.

flammation $(P>0.05, P=0.353)$. Foreign body reaction and granulation tissue were not detected in any of the groups. Also, degeneration in axon, myelin or perineural nets was not detected in any of the groups.

\section{DISCUSSION}

In general, $\mathrm{BC}$ consists of the exothermic reaction between poly acrylic acid or copolymer of acrylic acid/maleic acid liquid and aluminosilicate form of the glass-containing phosphate and fluoride. This reaction occurs rapidly (can be applied in 1-2 minutes) and results polymer-like structure that is formable and hardens in 5 minutes [15]. The glass structure in $\mathrm{BC}$ consists of three main structures, silica $\left(\mathrm{SiO}_{2}\right)$, alumina $\left(\mathrm{Al}_{2} \mathrm{O}_{3}\right)$ and calcium fluoride $\left(\mathrm{CaF}_{2}\right)$, and may include calcium oxide, aluminum phosphate and sodium aluminum fluoride $\left(\mathrm{Na}_{3} \mathrm{AlF}_{6}\right.$, cryolite), as well [15].

Currently, autografts, homografts and allografts are frequently used to repair middle ear ossicles. However, BC has been in- creasingly used for middle ear ossicular reconstruction. Many studies have reported successful auditory outcomes obtained with the reconstruction of the incudostapedial joint by $\mathrm{BC}$ [1$4,13]$. Since the use of $B C$ is becoming more widespread researches about BC is increasing gradually. Studies have shown that $\mathrm{BC}$ has cytotoxic effects on odontoblasts, milk teeth stem cells, mouse lymphoma cells, macrophages, fibroblasts, mouse embryonic stem cells, and dental pulp cells [6,16-21]. In literature, despite the reports of various side effects of $\mathrm{BC}$ on neurons, and neurotoxicity cases resulted in death, objective and evidence-based studies towards BC-related neurotoxicity are still inadequate. Neurotoxicity can be described as a neural death or impaired nervous system which is caused by toxic effects of a natural or artificial substance to the brain or peripheral nervous system [22]. Neurotoxic effect of BC is usually caused by aluminum ion release. In particular, in cases such as acoustic neuroma surgeries and cranial defect repairs, where high levels of $\mathrm{BC}$ are needed, fatal dura and brain tissue inflammation cases were reported due to exposure of cerebrovascular fluid to aluminum $[8,9,23]$. Renard et al. [8] reported subacute aluminum 
myoclonic encephalopathy in two patients. Reusche et al. [9] reported an aluminum encephalopathy case, which occurred after the use of $\mathrm{BC}$ for acoustic neuroma surgery. Following loss of consciousness and myoclonic seizures, patient died from sepsis on the postoperative 6 month. Patient's postoperative cerebrospinal fluid alumina level was increased, suggesting aluminum release after exposure of cerebrovascular fluid to $\mathrm{BC}$ which used for reconstruction. Postmortem analysis showed inclusion bodies contain aluminum in the plexus cells and cortex neurons. It has been reported that direct exposure of BC to neural tissue is contraindicate since it may cause aluminum release when exposed and should be handled carefully to avoid contact with the facial nerve and Jacobson neuron in the middle ear [10]. Granstrom et al. [7] reported complete facial paralysis after 3 weeks of $\mathrm{BC}$ use for reconstruction of the external ear canal. Six weeks after the clearance of $\mathrm{BC}$, facial paralysis recovered completely, suggesting that the paralysis caused by aluminum release.

In our study, we assessed neurotoxicity by evaluating possible nerve conduction problems, by measuring CMAP with evoked EMG (also-called electroneuronography). In electroneuronography (ENoG), CMAPs are measured by stimulating the facial nerve from stylomastoid foramen using bipolar stimulating electrode bilaterally with maximal electrical stimulation and the responses are recorded by the second bipolar electrode in several regions of facial nerve and the percentage of degeneration is calculated by comparing the responses of the two sides [24]. ENoG is not measured in the first 72 hours because 72 hours are required for Wallerian degeneration to reach the stylomastoid foramen from the intratemporal portion. On the 21st day after paralysis, Wallerian degeneration is completed and nerve excitability is lost. We planned measurements in our study to be preoperative, postoperative second and fourth weeks, but due to technical reasons we could not apply our second week measurements. Nonetheless, our fourth week measurements alone are valuable in terms of giving an idea. The use of ENoG between the first 72 hours and the 21st day, which is used to assess the prognosis of acute facial paralysis, is valuable in terms of the need for surgical intervention in acute facial paralysis. After day 21, the excitability of the nerve will disappear because Wallerian degeneration is complete. In our study, the ability to perform measurements at fourth weeks shows that all animals have intact facial nerves and no Wallerian degeneration. The absence of any pathological findings in the facial nerve in the histopathological examination supports this situation. The decline in the amplitudes suggests that peripheral muscle tissue is affected by aluminum toxicity. None of the animals demonstrated postoperative facial paralysis, dysregulation in oral intake or loss of activity in whiskers. While no significant difference was detected in BC-applied groups for latency, duration, and supramaximal stimuli values, there was a significant decrease in amplitude value for group $\mathrm{D}(P<0.05, P=0.043)$. No changes in the latency time indicate that there was no transmission block occurred, and nerve conduction has not been affected by application of BC. Degeneration of motor neuron, myopathy or neuromuscular motor junction diseases cause decreases in amplitude while latency stays stable [13]. Histopathological examination in our study showed that there was no degeneration in axon, myelin or perineural nets, which suggested possible problems in neuromuscular end plate or myopathy instead of axonal damages. In addition, two animals in group D had inflammation and myositis in muscular tissue and the decreases in their amplitude value could be related to myopathy. Results that indicate decreases in amplitude without any alterations in latency time, are similar to a thesis study of Kosar [13], which investigated the neurotoxici-
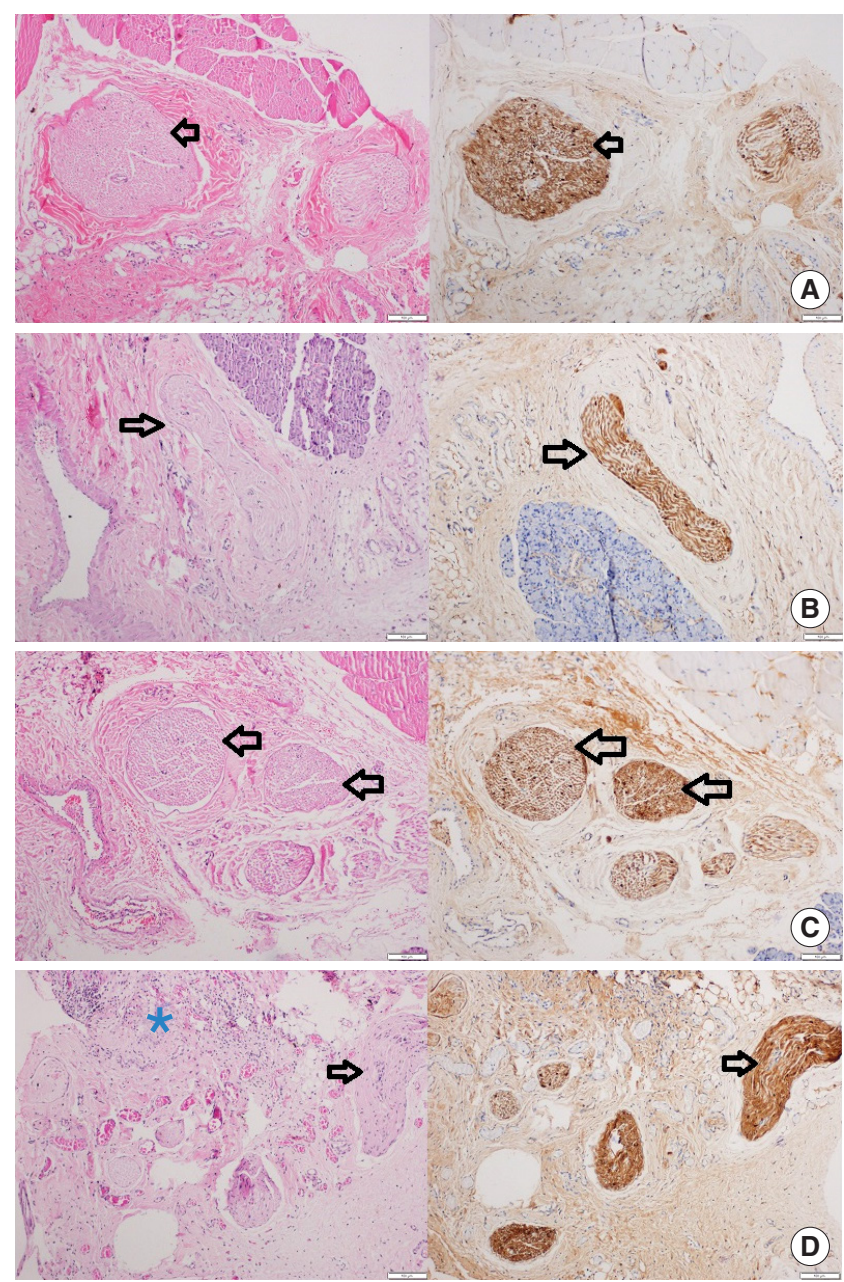

Fig. 2. Histopathologic section of the facial nerves and surrounding tissues of rats in all groups: H\&E and s-100 stain (left and right, respectively), scale bar indicates $100 \mu \mathrm{m}$. The section is from (A) group A (control), (B) group B (sham), (C) group C (bone cement $[B C]$ is applied for 5 seconds and cleaned), and (D) from group D (BC was applied and kept for 5 minutes drying). There was no axonal degeneration in all figures. Perineural sheath and myelin structure layout were also regular (shown with arrows), but there was an inflammation of muscle and myositis in a rat tissue of group D (shown with asterisk). 
ty of $\mathrm{BC}$ on the sciatic nerve of rabbits. The author [13] correlated this situation with inflammation and toxicity in muscular tissue; however, this correlation was unable to be confirmed due to the lack of muscular pathological examination. In our study, two pathology preparations in group $\mathrm{D}$ showed a presence of inflammation and myositis in the peripheral muscle tissue around the facial nerve (Fig. 2).

Righini-Grunder et al. [1] reported an inflammatory foreign body reaction in the $4.3 \%$ of the 444 patients which they used $\mathrm{BC}$ and they associated this inflammatory foreign body reactions with BC degradation. Kupperman and Tange [23] reported granulation formation and sequestration reactions in 23 cases of mastoid cavity obliteration with $\mathrm{BC}$, and they suggested that BC use has a high risk for rejection reactions. Bayazit et al. [25] reported a case of external ear canal reconstruction with $\mathrm{BC}$ and observed a formation of granulation tissue in the external auditory canal. Subsequently, Babighian [26] reported foreign body reaction in three cases of canal reconstruction in a total of 63 cases of ossicular repair, cochlear implant fixation, and external auditory reconstruction. In histopathological examination, we did not observe statistically significant difference between groups in terms of inflammation. Foreign body reaction and granulation tissue were not detected in any of the groups. Also, none of the groups demonstrated degeneration in axon, myelin or perineural nets.

In conclusion, to best of our knowledge, this is the first experimental study to investigate neurotoxic effects of $\mathrm{BC}$ on facial nerve by using electrophysiological and histopathological methods. None of the animals demonstrated facial paralysis and there were not any detectable nerve conduction blocks. Decrease of CMAP in group D could be associated with the inflammation of surrounding tissue and myositis. In histopathological examination, direct exposure of $\mathrm{BC}$ to neurons did not induce degeneration in axon, myelin or perineural nets, but also minimal inflammation and myositis were observed. Our study results suggest that $\mathrm{BC}$ has no direct toxicity on facial nerve, while it has indirect effects, by decreasing amplitude. However, further, large-scale studies are required to confirm these findings.

\section{CONFLICT OF INTEREST}

No potential conflict of interest relevant to this article was reported.

\section{ACKNOWLEDGMENTS}

This study was presented on 38th Turkish National Congress of Otorhinolaryngology-Head and Neck Surgery in Antalya Titanic Deluxe Belek Hotel on 26-30 October, 2016, Antalya, Turkey and 5th International Conference and Exhibition Surgery and ENT, 7-8 November, 2016, Alicante, Spain.

\section{REFERENCES}

1. Righini-Grunder F, Hausler R, Chongvisal S, Caversaccio M. Glass ionomer cement in otological microsurgery: experience over 16 years. Eur Arch Otorhinolaryngol. 2015 Oct;272(10):2749-54.

2. Hafiz G. A more reliable method for incudostapedial rebridging ossiculoplasty: bone cement and wire. Adv Ther. 2005 Jan-Feb;22(1): 56-62.

3. Kalcioglu MT, Tan M, Fleerakkers J.The use of bone cement for ossicular chain defects. Eur Arch Otorhinolaryngol. 2013 Nov;270(11): 2849-55.

4. Kalcioglu MT, Uzun IH, Yalcin M, Malkoc MA, Ogreten AT, Hanege FM. Evaluation on shear bond strength of different glass ionomer and hydroxy apatite cements used in ossiculoplasty. Balkan Med J. $2015 \mathrm{Jan} ; 32(1): 23-9$.

5. Guillard O, Pineau A, Fauconneau B, Chobaut JC, Desaulty A, Angot A, et al. Biological levels of aluminium after use of aluminium-containing bone cement in post-otoneurosurgery. JTrace Elem Med Biol. 1997 Apr;11(1):53-6.

6. Kanjevac TV, Milovanovic MZ, Milosevic-Djordjevic O, Tesic Z, Ivanovic M, Lukic A. Cytotoxicity of glass ionomer cement on human exfoliated deciduous teeth stem cells correlates with released fluoride, strontium and aluminum ion concentrations. Arch Biol Sci. 2015; 67(2):619-30.

7. Granstrom G, Holmquist J, Tjellstrom A. Facial nerve paralysis following repair of the external ear canal with ionomeric cement. Ear Nose Throat J. 2000 Jul;79(7):495-8.

8. Renard JL, Felten D, Bequet D. Post-otoneurosurgery aluminium encephalopathy. Lancet. 1994 Jul;344(8914):63-4.

9. Reusche E, Pilz P, Oberascher G, Lindner B, Egensperger R, Gloeckner K, et al. Subacute fatal aluminum encephalopathy after reconstructive otoneurosurgery: a case report. Hum Pathol. 2001 Oct; 32(10):1136-40.

10. Brook IM, Hatton PV. Glass-ionomers: bioactive implant materials. Biomaterials. 1998 Mar;19(6):565-71.

11. Hatton PV, Hurrell-Gillingham K, Brook IM. Biocompatibility of glass-ionomer bone cements. J Dent. 2006 Sep;34(8):598-601.

12. Murai N, Oda Y, Nagata I, Takahashi JA, Ishikawa M, Kikuchi H, et al. Neurotoxicity testing of a new bioactive bone cement. Neurol Med Chir (Tokyo). 1997 Feb;37(2):201-4.

13. Kosar AT. Neurotoxic effect of glass ionomer cement: experimental study. Medical speciality thesis [Internet]. Istanbul: The Ministry of Health of Turkey, Sisli Etfal Training and Research Hospital, Department of Otorhinolaryngology; 2008 [cited 2018 Mar 1]. Available from: http://www.istanbulsaglik.gov.tr/w/tez/pdf/kbb/dr_alper_tolga_kosar.pdf.

14. Salomone R, Costa HJ, Rodrigues JR, Reis e Silva SM, Ovando PC, Bento RF. Assessment of a neurophysiological model of the mandibular branch of the facial nerve in rats by electromyography. Ann Otol Rhinol Laryngol. 2012 Mar;121(3):179-84.

15. Nicholson JW, Czarnecka B. Review paper: role of aluminum in glass-ionomer dental cements and its biological effects. J Biomater Appl. 2009 Nov;24(4):293-308.

16. Aranha AM, Giro EM, Souza PP, Hebling J, de Souza Costa CA. Effect of curing regime on the cytotoxicity of resin-modified glass-ionomer lining cements applied to an odontoblast-cell line. Dent Mater. 2006 Sep;22(9):864-9.

17. Ribeiro DA, Marques ME, Salvadori DM. Biocompatibility of glass- 
ionomer cements using mouse lymphoma cells in vitro. J Oral Rehabil. 2006 Dec;33(12):912-7.

18. Meryon SD, Stephens PG, Browne RM.A comparison of the in vitro cytotoxicity of two glass-ionomer cements. J Dent Res. 1983 Jun; 62(6):769-73.

19. Kong N, Jiang T, Zhou Z, Fu J. Cytotoxicity of polymerized resin cements on human dental pulp cells in vitro. Dent Mater. 2009 Nov; 25(11):1371-5.

20. Souza PP, Aranha AM, Hebling J, Giro EM, Costa CA. In vitro cytotoxicity and in vivo biocompatibility of contemporary resin-modified glass-ionomer cements. Dent Mater. 2006 Sep;22(9):838-44.

21. Nguyen Ngoc TD, Son YO, Lim SS, Shi X, Kim JG, Heo JS, et al. Sodium fluoride induces apoptosis in mouse embryonic stem cells through ROS-dependent and caspase- and JNK-mediated pathways.
Toxicol Appl Pharmacol. 2012 Mar;259(3):329-37.

22. Ramsden RT, Herdman RC, Lye RH. Ionomeric bone cement in neuro-otological surgery. J Laryngol Otol. 1992 Nov;106(11):949-53.

23. Kupperman D, Tange RA. Ionomeric cement in the human middle ear cavity: long-term results of 23 cases. Laryngoscope. 2001 Feb; 111(2):306-9.

24. Lee DH. Clinical efficacy of electroneurography in acute facial paralysis. J Audiol Otol. 2016 Apr;20(1):8-12.

25. Bayazit YA, Ozer E, Kanlikama M, Durmaz T, Yilmaz M. Bone cement ossiculoplasty: incus to stapes versus malleus to stapes cement bridge. Otol Neurotol. 2005 May;26(3):364-7.

26. Babighian $G$. Use of a glass ionomer cement in otological surgery: a preliminary report. J Laryngol Otol. 1992 Nov;106(11):954-9. 